\title{
Neuromorphic Adaptable Ocular Dominance Maps
}

\author{
Priti Gupta, Mukti Bansal, and C.M. Markan \\ VLSI Design Technology Lab \\ Department of Physics \& Computer Science \\ Dayalbagh Educational Institute \\ AGRA - 282005 \\ markan_cm@hotmail.com
}

\begin{abstract}
Time staggered winner-take-all (ts-WTA) is a novel analog CMOS neuron cell [8], that computes 'sum of weighted inputs" implemented as floating gate pFET 'synapses'. The cell behavior exhibits competitive learning (WTA) so as to refine its weights in response to stimulation by input patterns staggered over time such that at the end of learning, the cell's response favors one input pattern over others to exhibit feature selectivity. In this paper we study the applicability of this cell to form feature specific clusters and show how an array of these cells when connected through an RC-network, interacts diffusively so as to form clusters similar to those observed in cortical ocular dominance maps. Adaptive feature maps is a mechanism by which nature optimize its resources so as to have greater acuity for more abundant features. Neuromorphic feature maps can help design generic machines that can emulate this adaptive behavior.
\end{abstract}

Keywords: Floating Gate pFET, competitive learning, WTA, Feature maps, ocular dominance.

\section{Introduction}

Interconnectivity patterns between hierarchically organized cortical layers are known to extract different sensory features from a sensory image and map them over the cortical sheet. Higher cortical layers successively extract more complex features from less complex ones represented by lower layers. In fact it has been shown that different sensory cortices are also an outcome of a mechanism by which a generic cortical lobe adapts to the nature of stimulus it receives so as to extract sensory features embedded in it. [1]. Thus feature extraction and hence formations of feature maps are fundamental underlying principles of parallel and distributed organization of information in the cortex. Any effort towards an artificial or neuromorphic realization of cortical structure will have to comprehend these basic principles before any attempt is made to derive full benefit of cognitive algorithms that are active in the brain. Neuromorphic realization of cortical map finds useful application in the area of robotic vision where potential improvement are possible by employing mechanisms observed in a living brain [2]. Dedicated adaptive hardware can help design generic machines that conserve resources by acquiring greater sensory acuity to more abundant features at the cost of others 
depending on the environment they are nurtured. Another emerging area of interest is that of neural prostheses wherein implants such as retinal or cochlear artificially stimulate sensory nerves to overcome blindness or deafness. Such implants are effective only when cortical infrastructure (feature maps) to interpret inputs from these implants is intact [3]. Animals born with defunct sensory transducers find their representative cortical area encroached upon by competing active senses. In such animals or in those who have a damaged or diseased cortex, sensory implants are ineffective as cortical apparatus (maps) to interpret inputs from these implants is not in place. The only option in such cases is to revive the cortical feature maps either biologically or through prosthetic neuromorphic realization [3].

In this paper we build a framework for adaptive neurmorphic cortical feature maps. In section 2 we present a basic building block for competitive learning (ts-WTA). In section 3 we apply $t s$-WTA to extract visual features e.g. ocular dominance and investigate its ability to cluster on the basis of their feature preference i.e. form feature maps.

\section{2 'Time Staggered WTA' Circuit}

In building adaptive neuromorphic structures, the biggest bottleneck has always been implementing adaptable connection strengths (weights) in hardware. While capacitor storage is volatile, the non-volatile digital storage is bugged with severe overheads of analog/digital domain crossing. However, improved quantum mechanical charge transfer processes across the thin oxide now offer a floating gate pFET whose threshold voltage and hence conductivity adapts much like Hebbian learning in a 'synapse' [4]. In fact, recently a competitive learning rule similar to Kohonen's unsupervised learning was implemented using floating gate pFETs [5].

\subsection{Floating Gate 'Synapses'}

The trapped charge on the floating gate of a pFET is altered using two antagonistic quantum mechanical transfer processes [4]:

Tunneling: Charge can be added to the floating gate by removing electrons from it by means of Fowler-Nordheim tunneling across oxide capacitor. The tunnel current is expressed in terms of terminal voltages across oxide capacitor i.e. tunnel voltage $V_{T}$, and floating gate voltage $\mathrm{V}_{\mathrm{fg}}$ as [6]

$$
I_{\text {tunnel }}=F_{T}\left(V_{T}, V_{f g}\right)=I_{t o} \exp \left(-\frac{V_{f}}{V_{T}-V_{f g}}\right)
$$

Injection: Charge is removed from the floating gate by adding electrons to it by current hot-electron injection (IHEI) from channel to the floating gate across the thin gate oxide. The injection current is expressed as a semi-empirical relationship in terms of pFET terminal voltages i.e. source $\left(\mathrm{V}_{\mathrm{s}}\right)$, drain $\left(\mathrm{V}_{\mathrm{d}}\right)$, floating gate voltage $\left(\mathrm{V}_{\mathrm{fg}}\right)$ and source current $\mathrm{I}_{\mathrm{s}}$ as [6]

$$
I_{\text {injection }}=F_{I}\left(V_{d}, I s, V_{f g}\right)=\eta \cdot I s \cdot \exp \left(-\frac{\beta}{\left(V_{f g}-V_{d}+\delta\right)^{2}}+\lambda \cdot V_{s d}\right)
$$



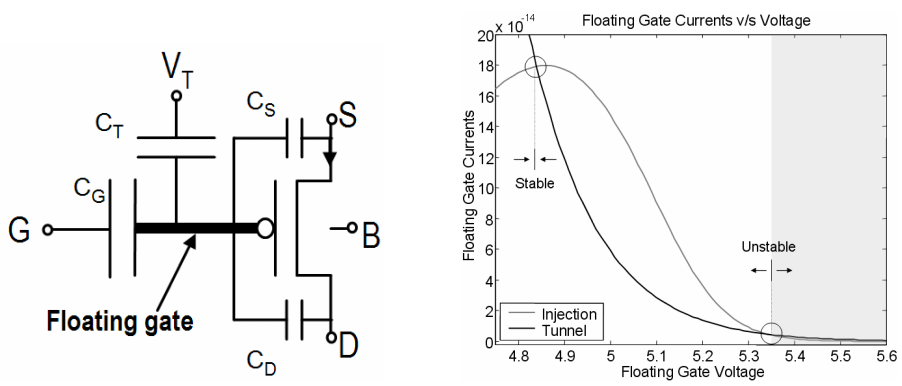

Fig. 1. (Left) A floating gate pFET, (right) Variation of Injection and Tunnel current as a function of Vfg for fixed tunnel voltage VT and drain voltage Vd

With floating gate capacitatively linked to input gate, drain, tunnel and source terminals of pFET (see figure 1(left)), the overall effect of continuous floating gate currents is expressed using Kirchoff's Current Law at the floating gate node:

$$
C_{F} \frac{\partial V_{f g}}{\partial t}=C_{G} \frac{\partial V_{G}}{\partial t}+C_{D} \frac{\partial V_{D}}{\partial t}+C_{T} \frac{\partial V_{T}}{\partial t}+C_{S} \frac{\partial V_{S}}{\partial t}+I_{\text {tunnel }}-I_{\text {injection }}
$$

\subsection{Adaptation in Floating Gate 'Synapses'}

Keeping $V_{G}$ constant and variation in $V_{S}$ small and by increasing the capacitances at the drain terminal, the variation of charge on floating gate can be reduced to

$$
C_{F} \frac{\partial V_{f g}}{\partial t}=I_{\text {tunnel }}-I_{\text {injection }}=F_{T}\left(V_{T}, V_{f g}\right)-F_{I}\left(V_{d}, I_{s}, V_{f g}\right)
$$

With pFET in saturation its source current is largely expressed as a function of $V_{f g}$, so that above equation is rewritten with magnitude of $I_{s}$ absorbed in $F_{I}$

$$
C_{F} \frac{\partial V_{f g}}{\partial t}=I_{\text {tunnel }}-I_{\text {injection }}=F_{T}\left(V_{T}, V_{f g}\right)-F_{I}\left(V_{d}, V_{f g}\right) . x
$$

where binary $x$ is 1 when $I_{s}$ current flows and 0 otherwise.

Variation of $I_{\text {tunnel }}$ and $I_{\text {injection }}$ is plotted as a function $V_{f g}$ in figure 1(right). Two salient points (i) stable and (ii) unstable point characterize the adaptation dynamics of floating gate $\mathrm{pFET}$. $\mathrm{V}_{\mathrm{fg}}$ above unstable point increases uncontrollably as $\mathrm{I}_{\text {tunnel }}>$ $\mathrm{I}_{\text {injection. }}$. Below unstable point, $\mathrm{V}_{\mathrm{fg}}$ always gravitates towards the stable point. Any variation in $\mathrm{V}_{\mathrm{T}}$ and $\mathrm{V}_{\mathrm{d}}$ merely relocates both stable and unstable point, though latter is affected less substantially. Thus we have a 'synapse' with an adaptable conductivity or weight which grows on stimulation (injection) and saturates to an upper limit (stable point). In absence of stimulation the self decay term (tunneling) prunes its weights till it finally goes dead from where it cannot recover (unstable point).

\section{3 'Time Staggered Winner Take All'}

We now build a competitive learning cell by connecting two such weighted inputs or 'synapses' as shown in figure 2(left) such that the voltage at common source terminal 

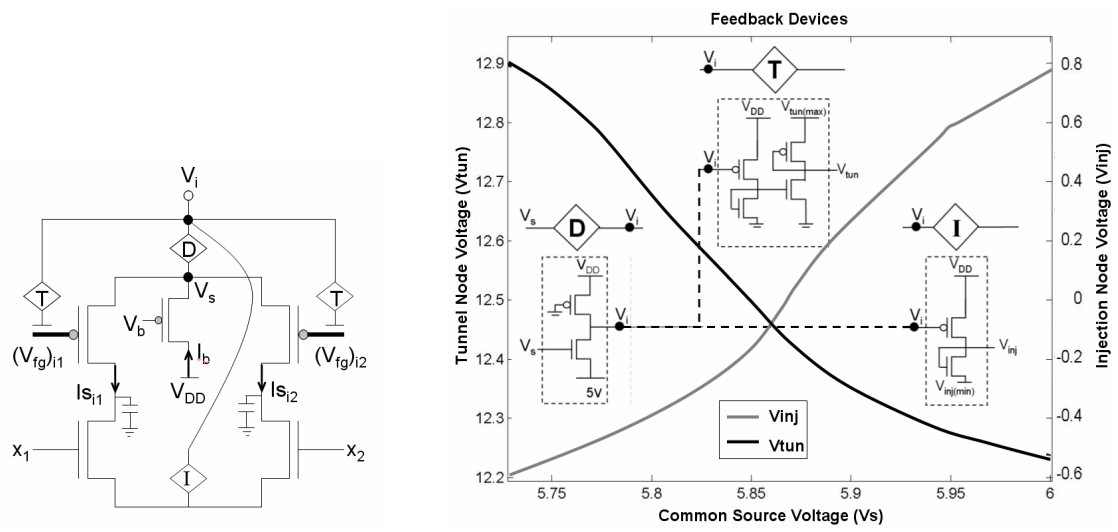

Fig. 2. (Left) Actual circuit of learning cell, $\left(\mathrm{V}_{\mathrm{fg}}\right)_{\mathrm{i} 1},\left(\mathrm{~V}_{\mathrm{fg}}\right)_{\mathrm{i} 2}$, shows the floating gate based weighted connection. I, T and D are dependent voltage sources for injection, tunnel and activation node. $\mathrm{x}_{1}, \mathrm{x}_{2}$ are inputs and node voltage $\mathrm{V}_{\mathrm{S}}$ is common source voltage of the cell. (Right) Shows Feedback devices T(green), I(blue). Both the devices act in conjunction with buffer device ' $D$ ' and their equivalent circuits are shown as inset. The graph shows variation of common tunnel node voltage $\mathrm{V}_{\text {tun }}$ and common Injection node $\mathrm{V}_{\text {inj }}$ w.r.t. $\mathrm{V}_{\mathrm{S}}$.

$\left(V_{s}\right)$ is expressed as a weighted sum of the two branch currents, $V_{S}=V_{D D}-I_{b} \cdot R_{b}$ where $R_{b}$ is the saturation resistance of pFET with a fixed bias voltage $V_{b}$, and source current $\mathrm{I}_{\mathrm{b}}$. With $x_{j}=1(0)=>j^{\text {th }}$ branch $\mathrm{nFET}$ is $\mathrm{ON}(\mathrm{OFF})$, i.e. $\mathrm{V}_{\text {gate }}=\mathrm{V}_{\mathrm{DD}}(0)$ we write

$$
\begin{gathered}
I_{b_{i}}=\sum_{j} I s_{i j} \cdot x_{j}=I s_{i 1} \cdot x_{1}+I s_{i 2} \cdot x_{2} \\
V_{s}=\left[V_{D D}-R_{b} \cdot \sum_{j} I s_{i j} \cdot x_{j}\right]
\end{gathered}
$$

Assuming floating gate pFET is in saturation, source current largely depends on its gate-to-source voltage. Also with one input applied at a time, source voltage $\left(\mathrm{V}_{\mathrm{s}}\right)$ itself depends on floating gate voltage of stimulated branch and so it won't be improper to express source current as a function of floating gate voltage i.e. $I s_{i j}=f\left(V_{f g, i j}\right) \cdot x_{j}$. Rewriting $(5)$, after substitution and suppressing constants, we have

$$
V_{s}=\left[\sum_{j} f\left(V_{f g}\right)_{i j} \cdot x_{j}\right]
$$

\subsection{Feedback}

Let us express tunnel feedback $V_{T}=T\left(V_{i}\right)$ and Injection feedback $V_{d}=I\left(V_{i}\right)$ as monotonically varying functions of activation node voltage $V_{i}$ which itself is linked to common source voltage $\mathrm{V}_{\mathrm{s}}$ through a buffer device $\mathrm{D}$ as $\mathrm{V}_{\mathrm{i}}=\mathrm{D}\left(\mathrm{V}_{\mathrm{s}}\right)$ see figure 2. In our design of feature selective cell we are guided by the basic principle "to accomplish WTA a self-excitation (injection) must accompany global nonlinear inhibition (tunneling)" [7]. 
Rewriting adaptation eqn (3) after substitution and suppressing the constant terms

$$
\frac{d\left(V_{f g}\right)_{i j}}{d t}=F_{T}\left[T\left(\sum_{j} f\left(V_{f g}\right)_{i j} x_{j}\right),\left(V_{f g}\right)_{i j}\right]-F_{I}\left[I\left(\sum_{j} f\left(V_{f g}\right)_{i j} x_{j}\right),\left(V_{f g}\right)_{i j}\right] x_{j}
$$

With two inputs stimulated one at a time, there are two input patterns $\mathbf{x}_{1}: \mathrm{x}_{1}=1 \& \mathrm{x}_{2}=0$ or $\mathbf{x}_{2}: \mathrm{x}_{1}=0 \& \mathrm{x}_{2}=1$. If in every epoch all patterns occur once in random order (random-inside-epoch) then at the end of an epoch the adaptation rate of $\mathrm{V}_{\mathrm{fg}}$ will be

$$
\frac{d\left(V_{f g}\right)_{i 1}}{d t}=\left.\frac{d\left(V_{f g}\right)_{i 1}}{d t}\right|_{x_{1}}+\left.\frac{d\left(V_{f g}\right)_{i 1}}{d t}\right|_{x_{2}} ; \text { and } \frac{d\left(V_{f g}\right)_{i 2}}{d t}=\left.\frac{d\left(V_{f g}\right)_{i 2}}{d t}\right|_{x_{1}}+\left.\frac{d\left(V_{f g}\right)_{i 2}}{d t}\right|_{x_{2}} ;
$$

In order to achieve WTA it would be necessary that adaptation rates of the two floating gates, in the above equation, are of opposite sign. This is achieved by ensuring that overall gain of our feedback devices is greater than unity [8]. Initially if $\left(\mathrm{V}_{\mathrm{fg}}\right)_{\mathrm{i} 1}>\left(\mathrm{V}_{\mathrm{fg}}\right)_{\mathrm{i} 2}$ and the two arms are stimulated with equal probability i.e. as randominside-epoch[8] then this circuit will iteratively amplify the difference $\Delta\left(\mathrm{V}_{\mathrm{fg}}\right)$ so that $\left(\mathrm{V}_{\mathrm{fg}}\right)_{\mathrm{i} 1}$ rises to eventually switch off its pFET while $\left(\mathrm{V}_{\mathrm{fg}}\right)_{\mathrm{i} 2}$ falls to reach the stable point so that its pFET stays on. Adaptability in floating gates with time is shown in the figure 3.

Though our circuit, which we call as time-staggered WTA (ts-WTA), is topologically similar to Lazzaro's WTA(l-WTA) circuit [9][10], yet it has some subtle and fundamental differences. $l$-WTA achieves positive feedback through common source terminal keeping gate voltages constant. In $t s$-WTA +ve feedback is achieved through special devices (I, T) that act on floating gates to achieve WTA. In $l$-WTA positive feedback through common source terminal require all inputs be stimulated simultaneously and the competition is instantaneous, whereas $t s$-WTA requires stimulation of one input at a time and competition is staggered over several epochs. For clustering in self-organizing feature maps (SOFM), cells are required to communicate their feature selectivity through their output node. This cannot happen if all inputs are stimulated at the same time as required by $l$-WTA. Therefore for map formation it is necessary that inputs be applied one at a time over all the participating cells. This clearly distinguishes the importance of ' $t s$-WTA' w.r.t. $l$-WTA as a feature selective cell.
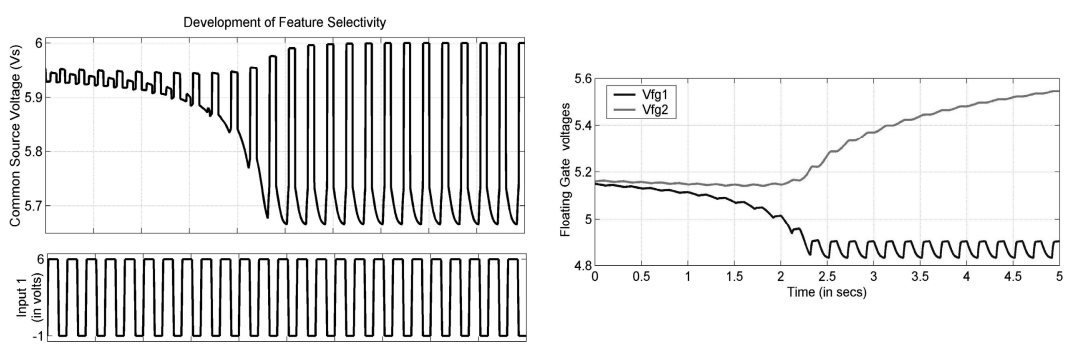

Fig. 3. (Left-top) Development of feature selectivity observed as response of the cell (Vs) to alternate input patterns. (Left-bottom) Input $\mathrm{x} 1, \mathrm{x} 2$ is invert of it. (Right) ts-WTA evolution of Vfgs. Here we have used alternate stimulation instead of random-inside-epoch. 


\section{Visual Feature Extraction}

Having described the basic competition learning cell as ts-WTA we now illustrate its applicability to one of the least complex of the visual features i.e. ocular dominance.

Cells of primary visual cortex of mammals have been observed to be dominated by inputs from one eye or the other, termed as ocular dominance. This domination is not genetically predetermined at birth rather develops in response to stimulus received from the two eyes. In animals whose one eye has been sutured or closed immediately after birth, cortical cells normally belonging to the closed eye are taken over or dominated by inputs from the active eye. This behavior is explained by the fact that at birth cortical cell receive nearly equal inputs from both eyes, slowly competition (similar to $t s$-WTA) between inputs from the two eyes leads to ocular domination. Thus ts-WTA cell can be directly used as ocular dominance feature selective cell provided its inputs represent inputs from the two eyes. At the end of learning, ts-WTA cell responds only to the input that wins the competition i.e. it is either left eye dominated or right eye dominated.

Map formation through diffusive interaction: Feature selectivity maps require that cells must cluster into groups with similar feature preferences such that there is both variety and periodicity in spatial arrangement of these cells across the cortical sheet. The basic mechanism that leads to clustering is based on the fact that neighboring cells have overlapping receptive fields and hence receive similar inputs. If somehow these neighboring cells can also be made to have similar responses, then because of Hebbian learning, individual cell's receptive field will also develop similarly. This similarity will extend over a portion of cortex where a majority of cells have similar initial feature biases. With randomly chosen initial feature biases, clusters of feature selective cells will emerge distributed over the cortical sheet similar to those observed in cortical feature selectivity maps.

Thus an important property a learning cell must posses to achieve clustering is the ability to develop its feature selectivity under influence of its neighbors. More explicitly, it should exhibit three types of adaptive behavior i.e. (i) if cell's bias is same as its neighbors, it must strengthen it, (ii) if cell's bias is opposite to its neighbors, it must reverse it, and (iii) if cell has equal number of neighbors with biases in favor and against, it must become unbiased i.e. respond equally to both inputs. Though theoretical models employ these principles in different ways, yet not all are easy to realize in hardware. A class of model that is relatively easier to implement are those based on Turing's reaction-diffusion framework. Biologically such an interaction occurs in the form of chemicals leaking out of an active cell which enter neighboring cells to lower their threshold thus encouraging them to also become active so that neighboring cells, with similar inputs and outputs, develop similar feature selectivity.

We model the diffusive interaction between feature selective cells by means of a RC network. Cells are connected to RC-grid through a diffusion device 'D', see figure 4. Device ' $\mathrm{D}$ ' serves (i) to isolate neighboring cells from directly altering activation of a cell, (ii) to drive the tunnel device that operates at higher voltage than voltages available at activation node. We test this hypothesis for two cases (i) a row of 10 learning cells that are diffusively coupled on a 1-D RC network, see 

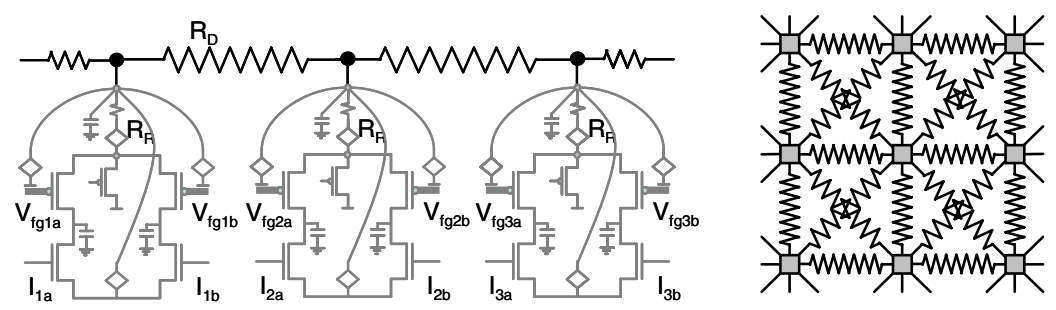

Fig. 4. Shows diffusive interaction between learning cells implemented in actual circuit by means of RC network. (left) 1-D, (right) 2-D, where every grey square represents ts-WTA cell.
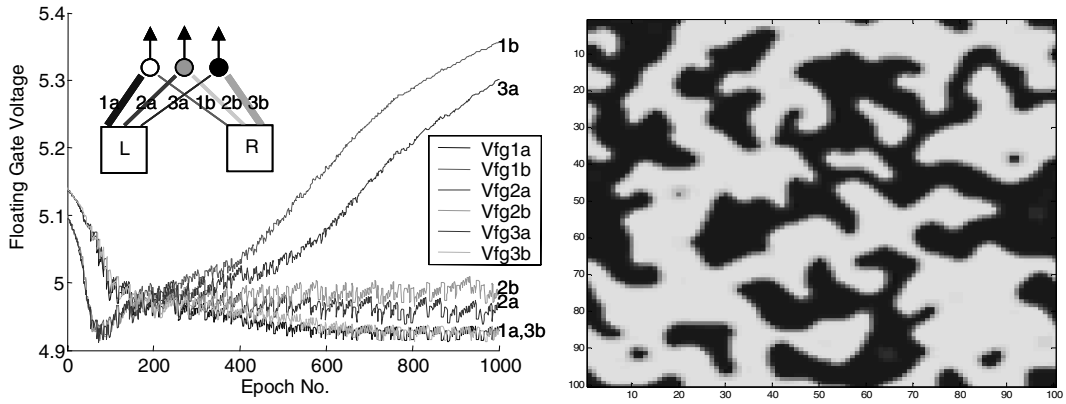

Fig. 5. (Left) Shows development of $\mathrm{V}_{\mathrm{FG}}$ for three cells of figure 4(left). (1) left eye dominated cell (white), (2) binocular cell (gray), (3) right eye dominated cell (black). Lower VFG implies stronger connection strength. A dominated cell develops to have a large difference between two VFGs, but a binocular cell has nearly equal VFGs. (Right) Simulation results for 2D array of 100x100 ts-WTA cells of ocular dominance. White (Black) represent cells dominated by left (right) eye, grey represents equal domination or binocular cell.

figure 4(left), and (ii) a 2-D RC grid of $100 \times 100$ cells, see figure 4(right). Here $R_{R}$ and $R_{D}$ refer to resistance values that balance the effect of Reaction term (activation of a cell to given input) and Diffusion term (effect of activation of neighboring cell) respectively. To avoid any boundary effects we take periodic boundary conditions. Results for 1-D are shown in Figure 5(left) and for 2-D in figure 5(right). Initially all cells are given faint random biases as either left or right dominated cells. During development all the cells are stimulated with identical patterns $\{(0,1),(1,0)\}$ in an order chosen randomly in every epoch. Under this random inside epoch stimulation cells perform 'time staggered WTA' i.e. strengthen their biases. Since these cells develop under influence of diffusive interaction they begin to clusters into left or right dominated cells, also cells which lie at the boundary of opposite biases turn binocular i.e. they respond equally to both left and right input. This is reflected in the Figure 5(right) as grey cells. The graph (figure 5(left)) shows three cells chosen such that cells at the two ends represent clusters of opposite biases and the cell in the centre favors one of them. After learning, cells at the two corners develop according to their own biases while the cell in the centre becomes unbiased with equal weights.

The pattern of ocular dominance produced with this array of RC-coupled ts-WTA cells is shown in figure 5 showing well distributed clusters of left (black) and right 
eye (white) dominated cells. Observe the smooth change from one cluster to other occurs due to development of grey (binocular) cells. The creation of clusters and existence of binocular cells are the major strengths of this diffusive-Hebbian model.

Acknowledgements. This work was funded by research grant to CMM, (III.6 (74)/99ST(PRU)) under SERC Robotics \& Manufacturing PAC, Department of Science and Technology, Govt. of India.

\section{References}

1. Horng, S.H., Sur, M.: Visual activity and cortical rewiring: activity-dependent plasticity of cortical networks. Progress in Brain Research 157, 3-11 (2006)

2. Choi, T.Y.W., et al.: Neuromorphic Implementation of Orientation Hypercolumns. IEEE Tran. on Circuit \& Systems-I 52(6), 1049-1060 (2005)

3. Merabet, L.B., et al.: What blindness can tell us about seeing again: merging neuroplasticity and neuroprostheses. Nature Reviews Neurosci. 6, 71-77 (2005)

4. Diorio, C., Hasler, P., Minch, B.A., Mead, C.A.: A Single-Transistor Silicon Synapse. IEEE Transactions on Electron Devices 43(11), 1972-1980 (1996)

5. Hsu, D., Figueroa, M., Diorio, C.: Competitive learning with floating-gate circuits. IEEE Transactions on Neural Networks 13(3), 732-744 (2002)

6. Rahimi, K., Diorio, C., Hernandez, C., Brockhausen, M.D.: A simulation model for floating gate MOS synapse transistors. In: ISCAS (2002)

7. Grossberg, S.: Adaptive pattern classification and universal recoding: I. Parallel development and coding of neural feature detectors. Biol. Cybern. 23, 121-134 (1988)

8. Bansal, M., Markan, C.M.: Floating gate Time staggered WTA for feature selectivity. In: Proc. of Workshop on Self-organizing Maps, WSOM 2003, Kitakyushu, Japan (2003)

9. Lazzaro, J., Ryckbusch, S., Mahowald, M.A., Mead, C.A.: Winner-take-all Networks of $\mathrm{O}(\mathrm{N})$ complexity, NIPS 1 Morgan Kaufman Publishers, San Mateo, CA, pp. 703-711 (1989)

10. Kruger, W., Hasler, P., Minch, B., Koch, C.: An adaptive WTA using floating gate technology. In: Advances in NIPS 9, pp. 713-719. MIT Press, Cambridge (1997) 